\title{
CORRESPONDENCE
}

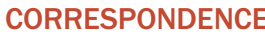

\section{Gated SPECT can detect abnormal RV structure or function}

\section{Mohammad R. Movahed}

In the Review by Luc L. Mertens and Mark K. Friedberg (Mertens, L. L. \& Friedberg, M. K. Imaging the right ventricle - current state of the art. Nat. Rev. Cardiol. 7, 551-563 (2010))1 describing imaging modalities applicable to the right ventricle, the role of gated single-photon emission computed tomography (SPECT) imaging was completely ignored and not mentioned at all. Cardiac SPECT imaging is one of the most commonly used cardiac tests in patients with chest pain. Gated SPECT imaging can be useful in patients with pulmonary embolism, an important life-threatening condition. Elevation of pulmonary arterial pressure in the setting of pulmonary embolism commonly leads to right ventricular (RV) pressure overload and a shift of the interventricular septum toward the left ventricle. Our group named this effect 'the Movahed's sign'. ${ }^{2-4}$ This sign can be easily recognized by paying attention to the septum during interpretation of gated SPECT imaging data, as it is revealed by the observation of a D-shaped left ventricle. Unfortunately, the right ventricle is commonly neglected during interpretation of gated SPECT imaging. ${ }^{4}$ Therefore, informing cardiologists about this relevant sign that can lead to a correct diagnosis of abnormal RV function is very important. In the first paper to report the Movahed's sign,
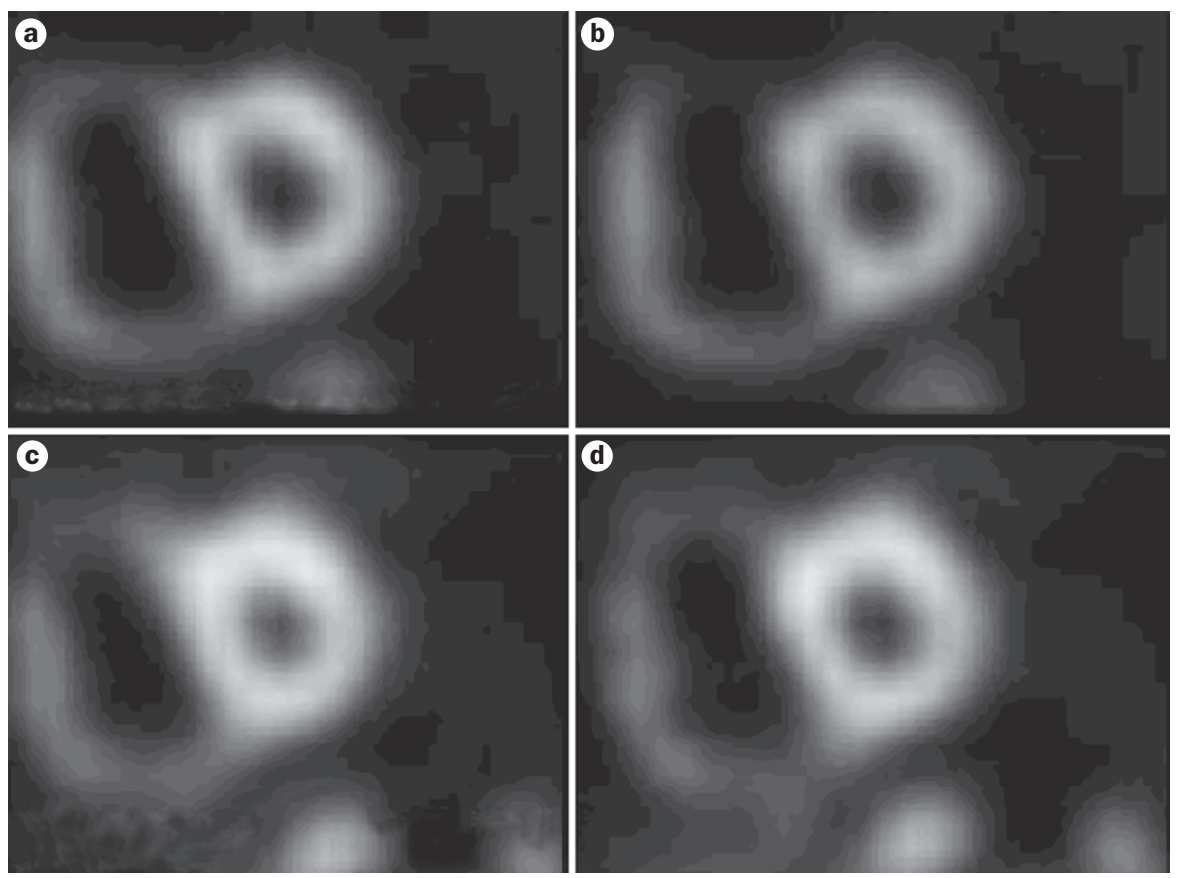

Figure 1 | D-shaped left ventricle, high right ventricular tracer uptake and increased right vernacular volume can be seen on routine gated SPECT imaging, and are findings consistent with right ventricular overload. The image shows short-axis views of slices of the heart of the same individual. $\mathbf{a} \mid$ and $\mathbf{b} \mid$ Stress images. $\mathbf{c} \mid$ and $\mathbf{d} \mid$ Resting images. Permission obtained from Movahed, M. R., Hepner, A., Lizotte, P. \& Milne, N. Flattening of the interventricular septum (D-shaped left ventricle) in addition to high right ventricular tracer uptake and increased right ventricular volume found on gated SPECT studies strongly correlated with right ventricular overload. J. Nucl. Cardiol. 12, 428-434 (2005). ${ }^{2}$

this imaging finding was more accurate than echocardiographic data in detecting a D-shaped left ventricle. ${ }^{2}$ The Movahed's sign has been now recognized by many authors to be useful in detecting the presence of abnormal RV function or status. ${ }^{5-7}$

In addition to the Movahed's sign, gated SPECT imaging provides other valuable ways to detect RV abnormalities, such as the ability to detect a high right-to-left ventricle tracer uptake ratio suggestive of pulmonary hypertension, ${ }^{6,8,9}$ or the visualization of the RV wall that enables recognition of an increased RV size suggestive of RV enlargement and overload. ${ }^{2-5,7,10}$ Under normal conditions, the right ventricle has $60 \%$ less myocardial tissue than the left ventricle, and, therefore, it has less total myocardial flow. Pulmonary hypertension or other conditions that lead to increased RV workload will raise $\mathrm{RV}$ myocardial flow, which is the main cause of increased RV uptake and RV size, both of which can be detected by gated SPECT imaging. ${ }^{11}$ Of note, relatively reduced left ventricular myocardial flow caused by myocardial infarction or threevessel coronary artery disease can also lead to relatively increased RV uptake, which needs to be considered in patients with high RV uptake seen during interpretation of gated SPECT imaging data. ${ }^{12}$ Figure 1 shows an example of a patient with pulmonary hypertension in whom gated SPECT imaging reveals the Movahed's sign, with a D-shaped left ventricle caused by septal flattening, together with high RV tracer uptake and increased RV volume.

Department of Medicine, Division of Cardiology, The Southern Arizona VA Health Care System, 3601 S $^{\text {th }}$ Avenue, Tucson, AZ 85723, USA rmova@aol.com

\section{doi:10.1038/nrcardio.2010.118-c1}

\section{Competing interests}

The author declares no competing interests.

1. Mertens, L. L. \& Friedberg, M. K. Imaging the right ventricle-current state of the art. Nat. Rev. Cardiol. 7, 551-563 (2010).

2. Movahed, M. R., Hepner, A., Lizotte, P. \& Milne, N. Flattening of the interventricular septum (D-shaped left ventricle) in addition to high right ventricular tracer uptake and increased right ventricular volume found on gated SPECT studies strongly correlates with right ventricular overload. J. Nucl. Cardiol. 12, 428-434 (2005). 
3. Murarka, S. \& Movahed, M. R. Review of Movahed's sign (D shaped left ventricle seen on gated SPECT) suggestive of right ventricular overload. Int. J. Cardiovasc. Imaging 26, 553-557 (2010).

4. Wackers, F. J. On the bright right side. J. Nucl. Cardiol. 12, 378-380 (2005).

5. Abikhzer, Y., Probst, S. \& Rush, C. Pulmonary hypertension findings detected by F-18 FDG PET scan. Clin. Nucl. Med. 33, 405-406 (2008).

6. Mazraeshahi, R. M., Striet, J., Oeltgen, R. C. \& Gerson, M. C. Myocardial SPECT images for diagnosis of pulmonary hypertension and right ventricular hypertrophy. J. Nucl. Med. Technol. 38, 175-180 (2010).

7. Shih, W. J., Kousa, K., Mitchell, B. \& Huang, W. S. Permanently increased brightness of right ventricle (D-shaped left ventricle) on myocardial perfusion imaging in a patient with chronic cor pulmonale: an autopsy correlation. J. Nucl. Cardiol. 13, 294-296 (2006).

8. Nakajima, K. et al. Estimation of right ventricular pressure by 201TI scintigraphy in paediatric cardiac disease. Nucl. Med. Commun. 11, 677-684 (1990).

9. Nakajima, K. et al. Assessment of right ventricular overload by a thallium-201 SPECT study in children with congenital heart disease. J. Nucl. Med. 32, 2215-2220 (1991).

10. Cohen, H. A. et al. Thallium 201 myocardial imaging in patients with pulmonary hypertension. Circulation 54, 790-795 (1976).

11. Wackers, F. J. et al. Pathophysiologic correlates of right ventricular thallium-201 uptake in a canine model. Circulation 64, 1256-1264 (1981).

12. Nestico, P. F., Hakki, A. H., Felsher, J., Heo, J. \& Iskandrian, A. S. Implications of abnormal right ventricular thallium uptake in acute myocardial infarction. Am. J. Cardiol. 58 , 230-234 (1986). 